\title{
Competencias tecnológicas de los docentes de ES y su relación con los instrumentos de evaluación para entornos virtuales - Contexto COVID-19.
}

\author{
Liliana Olivia Trixie Velazco Mareco \\ lilive0874@gmail.com \\ Facultad de Humanidades y Ciencias de la Educación \\ Universidad Nacional de Pilar
}

\section{RESUMEN}

La aparición del COVID-19 ha generado innumerables cambios en todos los ámbitos entre ellos requirió la inmediata incorporación de la tecnología en la Educación para la prosecución de las actividades donde el docente debió poner de manifiesto sus competencias tecnológicas para el desarrollo de las clases y aplicación de instrumentos de evaluación que evidencien el desarrollo de los procesos de enseñanza aprendizaje. En esta investigación se analiza la relación entre las competencias tecnológicas del docente y los instrumentos de evaluación para entornos virtuales desde una perspectiva relacionada a la Educación Superior, para el análisis se optó por la investigación bibliográfica, los materiales fueron obtenidos por medio del portal CICCO y de Google Académico. Los principales resultados indican que efectivamente existe un estrecho relacionamiento entre las competencias tecnológicas de los docentes para la aplicación efectiva de los instrumentos de evaluación del entorno virtual; en este contexto donde todo es virtual los instrumentos de evaluación deben proporcionar información que permita emitir juicios y tomar decisiones; las competencias de los docentes en tecnología facilitan la elaboración de instrumentos pertinentes además favorece la interacción entre el docente y el estudiante.

Palabras clave: Competencias tecnológicas del docente; Entorno virtual; Instrumentos de evaluación. 


\title{
Technological competencies of HE teachers and their relationship with assessment instruments for virtual environments - COVID-19 context
}

\begin{abstract}
The appearance of COVID-19 has generated innumerable changes in all areas, among them, it required the immediate incorporation of technology in Education for the continuation of activities where the teacher had to show his technological skills for the development of classes and application of assessment instruments that demonstrate the development of teaching-learning processes. In this research, the relationship between the technological competences of the teacher and the assessment instruments for virtual environments is analyzed from a perspective related to Higher Education, for the analysis we opted for bibliographic research, the materials were obtained through the CICCO portal and from Google Scholar. The main results indicate that there is indeed a close relationship between the technological competences of teachers for the effective application of the evaluation instruments of the virtual environment; In this context where everything is virtual, the assessment instruments must provide information that allows judgments and decisions to be made; Teachers' competencies in technology facilitate the development of pertinent instruments and also favor interaction between the teacher and the student.
\end{abstract}

Keywords: Technological competences of the teacher; Virtual environment; Evaluation instruments.

Artículo recibido: 03 oct. 2020 Aceptado para publicación: 14 nov. 2020 Correspondencia: lilive0874@gmail.com. Conflictos de Interés: Ninguna que declarar 


\section{INTRODUCCIÓN}

La pandemia de la COVID-19 generó un gran impacto a nivel mundial, en muchos lugares ha causado estragos en lo económico, educativo, salud y dentro del mismo contexto, problemas psicológicos y emocionales provocados por el confinamiento que fue una de las estrategias implementadas para combatir su propagación.

La Organización Mundial de la Salud (2020), define a la COVID-19 como "la enfermedad infecciosa causada por el coronavirus que se ha descubierto más recientemente. Tanto el nuevo virus como la enfermedad eran desconocidos antes de que estallara el brote en Wuhan (China) en diciembre de 2019”. En este sentido Brítez (2020), indica que causa enfermedades respiratorias, hasta el 6 de marzo ha matado a 74565 personas, con un total de 1345046 confirmados y 276515 recuperados según el portal tradingview.com. (p. 2).

Mendiola y otros (2020), mencionan que, específicamente en el ámbito de educación, obligó a la suspensión de clases en todos los niveles, con el confinamiento en casa de estudiantes y profesores, ha creado una red de efectos múltiples en todos los actores del proceso educativo. En el caso de las universidades, éstas han tenido que tomar una serie de medidas para acatar las indicaciones gubernamentales, al tiempo que intentan proporcionar a profesores y estudiantes con la infraestructura informática y tecnológica que les permita continuar con sus actividades académicas a distancia. Esta situación exigió implementar la modalidad de educación virtual, mediadas por las tecnologías del aprendizaje y el conocimiento, esta propuesta educativa evidenció las necesidades institucionales de infraestructura, sobre todo para repensar cómo se quiere que sea la transformación e implementación de la enseñanza, y la nueva cultura y práctica docentes. (p. 3).

Esta investigación bibliográfica busca describir los diferentes enfoques presentados por teóricos y los resultados de investigaciones sobre la relación existente entre los instrumentos de evaluación para entornos virtuales y las competencias tecnológicas del docente.

Para Camilloni, Celman, Litwin, y Palou de Maté, (1998), la cuestión de la evaluación relacionada al uso de tecnología y la comunicación no se refiere sólo a transferir modelos de evaluación tradicionales a entornos virtuales o analizar si es necesario generar nuevas concepciones relacionadas con los propósitos y formas de evaluación. El docente debe centrar su atención en la pertinencia de esos instrumentos y los fundamentos pedagógicos que sustentan una evaluación auténtica en los entornos virtuales. Deben tener en cuenta al 
momento de optar por alguno de ellos las oportunidades de aprendizaje y evaluación, qué aspectos se enriquecen o empobrecen la interacción entre docente y alumno. (pp. 6-7).

Camilloni (1998) también afirma que, el proceso de evaluar atraviesa los procesos de enseñanza y de aprendizaje de manera tal, que si se analizara y modificara en profundidad la idea de evaluar se modificarían sustancialmente los procesos de intervención de los docentes, y en el caso de los entornos virtuales de enseñanza aprendizaje, la implementación de las estrategias aplicadas. Citado en Lezcano y Vilanova, (2017, p. 2).

Siguiendo con el mismo planteamiento, sobre la importancia de la elección pertinente del instrumento para el contexto virtual, Dorrego, (2006), realiza una intervención al respecto mencionando que en muchos casos se les requiere a los docentes incluyan como técnicas de evaluación exámenes, test y otros similares, incluso en aquellos casos donde esto supone sacrificar la coherencia entre objetivos y resultados esperados o cuando se espera que los alumnos alcancen habilidades de pensamiento de alto nivel, pero luego la evaluación sólo enfoca niveles inferiores. Citado por Lezcano y Vilanova, (2017, p. 3).

Esta revisión se realizó, partiendo de la hipótesis de que existe un estrecho relacionamiento entre las competencias tecnológicas de los docentes para la aplicación efectiva de los instrumentos de evaluación del entorno virtual. Mediante apreciaciones y resultados de investigaciones, se realizó la contrastación de las mismas que facilitaron la realización de las conclusiones.

El conocimiento empírico pero vivenciado en el trabajo académico y activo durante esta pandemia de la autora, ha sido la fuente de motivación para la realización de esta investigación, mediante la indagación bibliográfica sustenta la hipótesis apoyada en resultados con tenor científico contextualizada a la población tomada como referencia, otra motivación fue la situación a la cual se han enfrentado las ES ante la necesidad de asumir el compromiso de proseguir las clases a pesar de las tantas dificultades que podrían presentarse; Cóndor-Herrera, (2020) destaca que cada sistema educativo debe tener claro que para que el modelo pedagógico asumido tenga éxito deben centrar sus acciones en superar tres aspectos fundamentales: La Implementación de recursos tecnológicos, capacitación del personal docente, adaptación de los estudiantes a esta forma de educación, sin olvidar que cada uno de estos aspectos deben enfocarse en buscar la calidad educativa. Dando por cierto que la primera acción para mitigar el impacto de la pandemia en la Educación es implementar una reingeniería organizacional, institucional, como siguiente 
paso se debe medir el impacto de la aplicación de esa estrategia; en este caso los receptores serán los alumnos; la pregunta es: ¿Qué herramientas de evaluación se aplican a los estudiantes?; si la prioridad es lograr una enseñanza de calidad, la interrogante debe ser esa; y es allí donde se puede indagar sobre ¿Qué tan contextualizados al entorno virtual están los instrumentos aplicados para evaluar?-

Barberá, (2006) menciona que la evaluación, implica la comunicación de criterios de evaluación, de los procesos y resultados, de las devoluciones que realiza el docente sobre la producción del estudiante, la evaluación es más que la consideración de los instrumentos y las evidencias relevadas. La comunicación, la interacción y la retroalimentación son las protagonistas; los instrumentos pueden ser facilitadores de estos procesos.

La siguiente problemática surge de la competencia de los docentes sobre la utilización de las TIC e instrumentos de evaluación sugeridos para entornos virtuales, García-Peñalvo, Abella-García, Corell, \& Grande, (2020) señalan: "Existe una brecha de competencias, relacionada con las competencias digitales del profesorado y del estudiantado para utilizar adecuadamente las plataformas digitales con fines educativos y la capacidad de crear o proveer contenidos y actividades educativas a través de estas", como se cita en MejíaFlores, Casquete-Baidal, Casquete-Baidal, \& Mackay-Castro, (2020, p. 1385).

Para llegar al objetivo propuesto, el trabajo se estructura de la siguiente forma, contiene una introducción con la descripción del propósito del estudio, la problemática que propicia el análisis y el estado del arte logrado mediante la revisión bibliográfica. Posteriormente se explica la metodología adoptada y la forma de acceso a los estudios científicos existentes en el tema. Se concluye con el resultado, discusión y conclusiones a las cuales se lograron llegar.

Los instrumentos de evaluación para entornos virtuales, es una de las variables estudiadas, al respecto Meriño Almaguer, Lorente Rodríguez y Maribona (2011), mencionan los resultados de la investigación de Ballena (2008), donde señala que los instrumentos de evaluación son formatos de registro de información que poseen particularidades propias basadas en el planteamiento de criterios e indicadores. Su naturaleza comprende la valoración del ejercicio que permite describir los propósitos de la evaluación mediante los indicadores. (p. 2).

Siguiendo a Meriño Almaguer, Lorente Rodríguez y Maribona (2011), encontramos que, con respecto a sus fines, toman lo expuesto por Depresbiteris, (2008), quien afirma que los 
instrumentos no son fines en sí mismos, pero constituyen una ayuda para obtener datos e informaciones del estudiante, por lo tanto, es necesario que el profesor ponga mucha atención en la calidad de estos ya que un instrumento inadecuado provoca una distorsión de la realidad. (p. 2).

Existen varios instrumentos que pueden ser empleados en los distintos tipos de enseñanza, entre ellos se pueden mencionar a las pruebas operatorias que tienen la finalidad de comprobar las habilidades de los estudiantes para operar con los contenidos aprendidos; el análisis y solución de casos es muy útil para el desarrollo de capacidades y habilidades del pensamiento; los mapas conceptuales utilizados para la presentación de las relaciones entre conceptos; los portafolios para la compilación de trabajos realizados por los estudiantes durante un período que evidencian el avance de los mismos en el proceso de aprendizaje. Los proyectos, las entrevistas, los diarios, las bitácoras, los debates, así como los talleres y los ensayos son algunos de los instrumentos más utilizados para evaluar a los estudiantes durante el proceso de enseñanza aprendizaje. (Meriño Almaguer, Lorente Rodríguez, y Maribona, 2011, p. 2).

El correcto empleo de los instrumentos permitirá tener información válida sobre el objeto seleccionado. Lezcano, (2016), señala que los juicios y decisiones que posteriormente se tomen dependen de la información brindada por ellos. Los instrumentos que se emplean para la etapa de relevamiento de información son parte del proceso de enseñanza y es allí donde cobran sentido, los mismos poseen un valor agregado como elementos facilitadores de la interacción entre docentes y estudiante. (p. 225).

Teniendo como marco de referencia principal la necesidad de adecuación de los instrumentos a un ambiente virtual; es oportuno considerar una apreciación realizada por Dillenbourg, Mendelsophn, y Jermann (1999), quienes señalan lo siguiente:

“... un ambiente de aprendizaje virtual puede definirse como un conjunto de herramientas integrado que permite la gestión del aprendizaje en línea, proporcionando un mecanismo de entrega, seguimiento de los estudiantes, la evaluación y el acceso a los recursos...”. (p. 8).

Inmersos en la vorágine del avance de la tecnología de la información y comunicación se han puesto en práctica nuevas formas de evaluar y varios son los instrumentos que contribuyen a mejorar este proceso. 
Entre la variedad de instrumentos de evaluación de aprendizajes en entornos virtuales: se pueden mencionar algunos como: pruebas objetivas, proyectos, rúbricas, creación de mapas conceptuales, foros, portfolios, wikis, etc. Algunos autores como Lara (2003), Arango (2004), Quesada (2006) Sanz (2008); Villar y Alegre (2013); proponen instrumentos de evaluación que pueden ser implementados dentro del entorno virtual, citado en Lezcano y Vilanova, (2017, p. 12).

Todo lo expuesto con antelación evidencia la necesidad de implementar instrumentos evaluativos pertinentes, que permitan conocer los resultados del proceso de enseñanza aprendizaje realizado por el docente, en contraparte, está la necesidad de que los mismos sean contextualizados y elaborados para un formato de entorno virtual.

La siguiente variable estudiada son las competencias tecnológicas del docente Universitario, para iniciar este análisis iniciamos un acercamiento desde la conceptualización de competencia docente; para Escudero (2006), es un conjunto de valores, creencias y compromisos, conocimientos, capacidades y actitudes que los docentes, tanto a título personal como colectivo (formando parte de grupos de trabajo e instituciones educativas) habrían de adquirir y en las que crecer para aportar su cuota de responsabilidad a garantizar una buena educación a todos (p. 34).

Afirmaciones realizadas por Unesco (1998); Perrenoud (2004); Zabalza, (2007); Imbernón (2006) plantean que, dentro de las competencias y características de un buen docente, se debe identificar el dominio de las nuevas tecnologías de la información y la comunicación como una de las competencias que deben poseer para el desempeño de su profesión. Hacen hincapié tanto en la consideración de las TIC como guías metodológicas y fuentes documentales, como en la necesidad de que los docentes dominen los nuevos avances en el proceso de enseñanza aprendizaje. Esto es lo que los habilita para poner en marcha procesos en modalidades presenciales o virtuales usando estos recursos didácticos como recursos complementarios o como un canal de comunicación y transmisión de información. Citados en Prendes Espinosa y Gutiérrez Porlán, (2013, p. 198).

Para poder definir el grado de competencia e individualizarlas, es necesario precisar criterios e indicadores, para ellos, una alternativa es considerar estándares, algo que sirve como norma, modelo o patrón de referencia. En este sentido, el Ministerio de Educación chileno (2006), realiza un aporte interesante en la que se apela a la necesidad de utilizar estándares como instrumento para que las instituciones encargadas de la formación docente 
puedan dar respuesta a las expectativas de la sociedad, un estándar sobre competencias TIC de los docentes lo cual definen como un patrón o modelo de referencia que permite, por una parte, determinar y valorar a aquellos docentes que son competentes en cuanto a dichas competencias y, por otra parte, orientar y guiar el diseño y la elaboración. Ministerio de Educación de Chile (2006). Citado en Prendes Espinosa y Gutiérrez Porlán (2013, p. 199). Siguiendo la misma línea de investigación, hallamos lo propuesto por la Unesco, (2008) la misma plantea que:

"Las nuevas tecnologías (TIC) exigen que los docentes desempeñen nuevas funciones y también requieren nuevas pedagogías y nuevos planteamientos en la formación docente. Lograr la integración de las TIC en el aula dependerá de la capacidad de los maestros para estructurar el ambiente de aprendizaje de forma no tradicional, fusionar las TIC con nuevas pedagogías y fomentar clases dinámicas en el plano social, estimulando la interacción cooperativa, el aprendizaje colaborativo y el trabajo en grupo". (p. 7).

Con respeto al tema, un aporte significativo y más objetivo lo realiza Prendes Espinosa y Gutiérrez Porlán (2013), quienes señalan que muchos países europeos cuentan con iniciativas nacionales en las que se recogen las habilidades que los docentes han de reunir en el campo de las TIC específicamente el Reino Unido, ya que actualmente es el país europeo que cuenta con estándares más detallados a este respecto. Los mencionados autores toman los estándares propuestos por la Unesco, (2004) donde plantean que los profesores han de estar capacitados para lo siguiente:

- Seleccionar y utilizar de forma apropiada una variedad de equipos y recursos tecnológicos con el objetivo de promover el aprendizaje.

- Ser usuarios competentes de una variedad de software y de herramientas tecnológicas y adaptarlas a la materia que enseñan y a la edad de sus alumnos.

- Examinar de forma crítica la relevancia del software y de las herramientas tecnológicas para las materias que enseñan y juzgar su valor potencial en su aplicación en la clase.

- Hacer uso constructivo de la tecnología de la información en sus clases y, en particular, preparar y poner en práctica planes de trabajo que incorporen de forma apropiada el uso de la tecnología.

- Evaluar las formas en que el uso de la tecnología produce cambios en la naturaleza de la enseñanza y el aprendizaje. (p. 201). 
Existen varios trabajos realizados sobre competencias TIC de los docentes, es oportuno destacar la propuesta del Ministerio de Educación de Chile (2006), que parte de la base de que en la sociedad actual se necesitan cada vez más y mejores docentes para responder a las demandas de esta, proponen que los docentes incorporen y aprovechen los recursos tecnológicos tanto en sus prácticas docentes como en su desarrollo profesional.

Las propuestas concretas se relacionan a las siguientes dimensiones que estructuran las competencias TIC:

a) Área pedagógica: los futuros docentes deben adquirir y demostrar formas de aplicar las TIC en el currículum vigente como una forma de apoyar y expandir el aprendizaje y la enseñanza.

b) Aspectos sociales, éticos y legales: los futuros docentes deben conocer, apropiarse y difundir entre sus estudiantes los aspectos éticos, legales y sociales relacionados con el uso de los recursos informáticos y contenidos disponibles en Internet, actuando de manera consciente y responsable respecto de los derechos, cuidados y respetos que deben considerarse en el uso de las TIC

c) Aspectos técnicos: los futuros docentes deben demostrar dominio de las competencias asociadas al conocimiento general de las TIC y del manejo de las herramientas de productividad (procesador de texto, hoja de cálculo, presentador) e Internet, desarrollando habilidades y destrezas para el aprendizaje permanente de hardware y software nuevos

d) Gestión escolar: los futuros docentes deben hacer uso de las TIC para apoyar su trabajo en el área administrativa, tanto por lo que hace a su gestión docente como para apoyar la gestión del establecimiento.

e) Desarrollo profesional: los futuros docentes deben hacer uso de las TIC como medio de especialización y desarrollo profesional, informándose y accediendo a diversas fuentes para mejorar sus prácticas y facilitando el intercambio de experiencias que contribuyan, mediante un proceso de reflexión con diversos actores educativos, a conseguir mejores procesos de enseñanza y aprendizaje. (Ministerio de Educación de Chile, 2006).

Algunos criterios e indicadores que podrían contribuir en la descripción del nivel de competencia TIC de los docentes son: 


\section{Conocimiento general sobre las posibilidades de las TIC. \\ - Conocimiento que poseen sobre el papel de las TIC en la futura profesión de sus alumnos. Posibilidades de las TIC para enriquecer su práctica docente. \\ - Conocimiento de buenas prácticas en el uso de TIC. \\ - Conocimiento de las políticas sobre TIC}

\section{Elección de recursos TIC para el aula.}

- Factores que llevan al docente a elegir un recurso TIC para el aula como, por ejemplo: que el recurso sea favorable para el aprendizaje del alumno, la motivación del alumnado o el grado de conocimiento del docente sobre el recurso.
Conocimiento y uso de estrategias metodológicas para el trabajo en red.

- Conocimiento sobre estrategias metodológicas para el trabajo en red más conocidas (trabajo cooperativo o colaborativo, el aprendizaje basado en proyectos, en estudios de casos o en pequeños grupos de discusión, el aprendizaje basado en problemas y los seminarios.)

- Conocimiento sobre las estrategias metodológicas menos conocidas (la caza del tesoro y la webquest)

\section{Conocimiento y uso de herramientas} telemáticas.

- Conocimiento sobre las herramientas de comunicación más conocidas como ser correo electrónico, foros y el chat. Las menos conocidas como el microblogging y los mundos virtuales.

- Otras herramientas podrían ser los buscadores, editores de texto, los creadores de presentaciones visuales, el software específico de cada ámbito de trabajo, editor multimedia, lifestreaming, los marcadores sociales, los lectores de RSS y las páginas de inicio personalizadas, plataformas de campus virtual.

Formación docente, investigación e innovación educativa con TIC.

- La realización de publicaciones de material didáctico en Internet. 
- La utilización de formatos abiertos en la publicación de contenidos y materiales didácticos en la red.

- Utilización de entornos de libre acceso para publicar la producción científica.

- Uso de las TIC para tareas docentes.

- Realización de tutorías electrónicas.

- Utilización de las TIC como herramientas de evaluación
- Participación en acciones formativas relacionadas con el uso de TIC.

- Participación en proyectos de investigación e innovación educativas con TIC.

- Acciones que llevan a cabo para mejorar sus competencias TIC (utilización de diferentes fuentes de información, plataformas y repositorios de recursos).

Fuente: Prendes Espinosa y Gutiérrez Porlán, (2013, pp. 213-216)

Por último, un aporte del trabajo realizado por Sánchez Mendiola y Martínez Hernández (2019), y publicado en el artículo "Retos educativos durante la pandemia de covid-19: una encuesta a profesores de la UNAM", enfatiza: "Esta investigación nos ayuda a comprender lo que viven los docentes y cómo podemos interpretarlo". En este sentido, el análisis e identificación de temas en las respuestas abiertas proporcionó información de gran utilidad para entender el reto al que se enfrentan y cómo puede la institución ayudarlos a solventarlos. La propuesta más importante de los docentes es la formación pedagógica, con todo lo que implica. Esto es fundamental ya que, como hemos comentado anteriormente, el proceso de desarrollo de los docentes es una de las tareas más importantes que debe promover la Universidad. Citado en Mendiola, y otros, (2020, p. 21).

\section{ESTRATEGIAS METODOLÓGICAS O MATERIALES Y MÉTODOS}

Para la realización de esta investigación bibliográfica se procedió a la revisión de artículos de diversos autores sobre el tema accediendo a ellos por medio de Google Académico y por el portal Cicco de Conacyt Paraguay, se realizó la búsqueda utilizándose las palabras clave: competencias docentes, instrumentos de evaluación para entornos virtuales. Se efectuó la revisión de algunas bibliografías de los años 2006 y 2008, posteriormente se dio énfasis en la búsqueda de materiales de los años 2016 hasta 2020, consecutivamente se procedió a la elaboración de fichas bibliográficas, en las mismas se asentaron datos de los materiales, año, título y objetivo del trabajo; se realizó el análisis exhaustivo de textos para 
incorporarlos a este trabajo considerando las fuentes, referencias de las mismas y las Normas de Estilo Apa.

Los criterios de inclusión considerados para la elección de los artículos estaban relacionados al logro del objetivo de esta investigación ya que existen muchas variables e innumerables teorías sobre competencias docentes e instrumentos de evaluación y la investigación requirió las relacionadas a las TIC y entornos virtuales; los años de publicación de los artículos y la confiabilidad de fuentes (artículos científicos publicadas por revistas indexadas y algunos trabajos de grado).

\section{RESULTADOS Y DISCUSIÓN}

Los resultados recabados mediante esta investigación sustentan en gran medida la relación existente entre las competencias tecnológicas del docente y los instrumentos de evaluación para entornos virtuales.

En primera instancia queda claro, que cada sistema educativo debe implementar un modelo pedagógico y centrar sus acciones en superar aspectos relacionados fundamentalmente con los recursos tecnológicos, capacitación del personal docente, adaptación de los estudiantes a esta forma de educación para el logro de una calidad educativa.

Con respecto al alcance y objetivo de los instrumentos de evaluación, se los define como formatos de registro de información que poseen particularidades propias basadas en el planteamiento de criterios e indicadores, la elección sobre la utilización de uno u otro queda a criterio del docente, los juicios y decisiones sobre los mismos depende de la información que se pueda recabar, de allí la importancia del mismo como componente de la interacción entre el docente y el estudiante.

Si bien la elección del instrumento queda a criterio del docente, como se mencionó en el párrafo anterior, la función de interacción que cumple es muy importante considerando el contexto actual, y la esencia de la práctica docente basada en ser el orientador y facilitador durante el proceso de enseñanza aprendizaje.

Varios autores plantean que, dentro de las competencias y características de un buen docente, está el dominio de las nuevas tecnologías de la información y la comunicación como una de las competencias que deben poseer para el desempeño de su profesión, esto lo habilita para poner en marcha procesos en modalidades presenciales o virtuales usando estos recursos didácticos como recursos complementarios o como un canal de comunicación y transmisión de información. 
Sobre el mismo tema, se propone que para lograr la integración de las TIC en el aula es necesario que el maestro estructure un ambiente de aprendizaje de forma no tradicional, fusionando las TIC con nuevas pedagogías incorporando clases dinámicas que estimulen la interacción en el proceso de enseñanza aprendizaje.

Específicamente García-Peñalvo, Abella-García, Corell, y Grande, (2020), menciona como resultado de su investigación que: "Existe una brecha de competencias, relacionada con las competencias digitales del profesorado y del estudiantado para utilizar adecuadamente las plataformas digitales con fines educativos y la capacidad de crear o proveer contenidos y actividades educativas".

Esta afirmación sustenta la relación existente entre las competencias tecnológicas de los docentes y su capacidad para proveer contenidos e instrumentos de evaluación.

Sobre la variedad de instrumentos de evaluación de aprendizajes en entornos virtuales: se mencionan los siguientes: pruebas objetivas, proyectos, rúbricas, creación de mapas conceptuales, foros, portfolios, etc.

\section{CONCLUSIÓN O CONSIDERACIONES FINALES}

La problemática presentada que da origen a este trabajo se basa el conocimiento empírico pero vivenciado en el trabajo académico y activo de la autora durante esta pandemia, que mediante la indagación bibliográfica sustenta su hipótesis de que existe un estrecho relacionamiento entre las competencias tecnológicas de los docentes para la aplicación efectiva de los instrumentos de evaluación del entorno virtual.

Se concluye que efectivamente existe una relación estrecha entre las competencias tecnológicas de los docentes y la implementación efectiva de los instrumentos de evaluación del entorno virtual, esto se afirma partiendo de la necesidad de que el docente cuente con competencias tecnológicas que contribuyan al mejor desenvolvimiento de su trabajo. Actualmente todo el sistema educativo ha proseguido las actividades por medio de sesiones virtuales utilizando plataformas o cualquier otro medio que facilite esa interacción. Si bien los instrumentos de evaluación mantienen sus formatos originales para clases presenciales con muy pocas variaciones para utilizar en entornos virtuales, los docentes deben adecuar los mismos y socializarlos, esta no es tarea fácil, para docentes con escaso conocimiento sobre las TIC, la única opción es utilizar los instrumentos que sean más fácil de redactar y en consecuencia muchos de ellos probablemente no cumplan con los criterios e indicadores básicos para la evaluación de competencias del alumno. 
También existen los docentes que son autodidactas para quienes esta tarea podría ser más llevadera, pero que también pone de manifiesto el escaso conocimiento.

Ante la verificación de esta hipótesis se puede deducir que urgen capacitaciones para los docentes, la prioridad es identificar sus competencias y partir de allí trabajar por subsanar cualquier debilidad.

Sánchez Mendiola y Martínez Hernández, (2019), señalan:

"Ante el reto que representa la pandemia y sus consecuencias económicas, psicológicas y sociales, una de las principales estrategias que debemos adoptar en las instituciones educativas es la formación y profesionalización en pedagogía y didáctica para la enseñanza y el aprendizaje universitarios, tomando en cuenta la infraestructura, las competencias y habilidades en tecnologías para el aprendizaje y el conocimiento."

Sobre el mismo punto, este autor señala: "Los testimonios de los docentes en cuanto a recursos tecnológicos, procesos institucionales, acompañamiento pedagógico y tecnológico, entre muchos otros, deben convertirse en fuertes llamadas de atención para las autoridades de las múltiples dependencias y entidades académicas que laboramos en la universidad". (Citado en Mendiola, y otros, 2020, p. 21).

\section{LISTA DE REFERENCIAS}

Barberá, E. (2006). Aportaciones de la tecnología a la eEvaluación. RED. Revista de Educación a Distancia (número especial VI). Obtenido de http://www.um.es/ead/red/M6

Brítez, M. (2020). La educación ante el avance del COVID-19 en Paraguay. Comparativo con países de la Triple Frontera. Preprint/Version 2, 1-15. Obtenido de https://preprints.scielo.org/index.php/scielo/preprint/view/22/579

Camilloni, A. R., Celman, S., Litwin, E., \& Palou de Maté, M. (1998). La evaluación de los aprendizajes en el debate didáctico contemporáneo. Buenos Aires: Paidós. Recuperado el 2020, de http://www.terras.edu.ar/biblioteca/12/ECPI_Camilloni_Unidad_3.pdf

Cóndor-Herrera, O. (2020). Educar en tiempos de COVID-19. CienciAmérica, 9(2), 3137.

Dillenbourg, P., Mendelsophn, P., \& Jermann, P. (1999). Why spatial metaphors are relevant to virtual campuses. En J. Levonen, \& J. Enkenberg, Learning and 
instruction in multiple contexts and settings. Bulletins of the Faculty of Education, 73. University of Joensuu, Finland, Faculty of Education.

Escudero, J. (2006). El profesor y su formación: Competencias y formación docente al servicio de un modelo de escuela en nuestro tiempo. En Programa y desarrollo temático de formación y actualización de la función pedagógica. Univeersidad de Muricia, 84-108.

García-Peñalvo, F. J., Corell, A., Abella-García, V., \& Grande, M. (2020). La evaluación online en la educación superior en tiempos de la COVID-19. eVSAL Revistas, 21. Obtenido de https://revistas.usal.es/index.php/eks/article/view/eks20202112

Lezcano, L. N. (2016). La evaluación de los aprendizajes en entornos virtuales desde la perspectiva del estudiante. Cibernética e Informática.

Lezcano, L., \& Vilanova, G. (Marzo de 2017). Instrumentos de evaluación de aprendizaje en entornos virtuales. Perspectiva de estudiantes y aportes de docentes. Unidad Académica Caleta Olivia.

Mendiola, M. S., Hernández, M., Carrasco, R. T., Servín, M., Hernández Romo, A. K., Jaimes Vergara, C. A., . . Rendón Cazales, V. J. (2020). Retos educativos durante la pandemia de covid-19: una encuesta a profesores de la UNAM. Revista Digital Universitaria.

Meriño Almaguer, Y., Lorente Roddríguez, A. E., \& Maribona, M. G. (2011). Propuesta de instrumentos de evaluación para entornos virtuales de aprendizaje: una experiencia en la universidad de las ciencias informáticas. Revista de Informática Educativa y Medios Audiovisuales, 8(15), 1-8.

Ministerio de Educación de Chile. (2006). Estándares en Tecnologías de la Información y la Comunicación para la Formación Inicial Docente. Obtenido de http://portal.enlaces.cl/portales/tp3197633a5s46/ documentos/2007071914 20080.Estandares.pdf

Organización Mundial de la Salud. (octubre de 2020). OMS. Obtenido de Preguntas y Respuestas sobre la enfermedad por coronavirus (COVID-19): www.who.int/es/emergencies/diseases/novel-coronavirus-2019/advice-forpublic/q-a-coronaviruses 
Prendes Espinosa, M. P., \& Gutiérrez Porlán, I. (Mayo-agosto de 2013). Competencias tecnológicas del profesorado en las universidades españolas. Revista de Educación, 196-222.

Sánchez Mendiola, M., \& Martínez Hernández, A. (2019). Formación Docente en la UNAM: Antecedentes y la Voz de su Profesorado. Coordinación de Desarrollo Educativo e Innovación Curricular, UNAM.

UNESCO. (2008). Estándares de competencias en TIC para docentes. Obtenido de http://www.eduteka.org/modulos/11/342/868/1 